\title{
Análisis psicométrico del cuestionario de memoria prospectiva y retrospectiva
}

\author{
Psychometric analysis of the prospective and retrospective
}

memory questionnaire

\author{
Evangelina Valeria Cores ${ }^{1}$, Melina Crespi $^{2}$, María Bárbara Eizaguirre ${ }^{3}$, \\ Ángeles Merino ${ }^{4}$, Cecilia Yastremiz ${ }^{5}$, Sandra Vanotti ${ }^{6}$ y Daniel Gustavo Politis ${ }^{7}$
}

${ }^{1}$ Doctora en Psicología. Investigadora Asistente del Consejo Nacional de Investigaciones Científicas y Técnicas (CONICET) en el Hospital Interzonal General de Agudos (HIGA) Eva Perón, San Martín, Buenos Aires. Jefa de trabajos prácticos de la cátedra de Neuropsicología, Facultad de Psicología, Universidad de Buenos Aires (UBA), Argentina. E-mail: evcores@psi.uba.ar ${ }^{2}$ Magister en Psicología Social Comunitaria. Profesora Adjunta en la Facultad de Psicología (UBA), Argentina. E-mail: melinacrespi@yahoo.com.ar

${ }^{3}$ Licenciada en Psicología. Neuropsicóloga en la Clínica de Esclerosis Múltiple, Centro Universitario de Neurología del Hospital Dr. J.M. Ramos Mejía. Facultad de Medicina, UBA. Jefa de trabajos prácticos de la Práctica Profesional Área Clínica, Neuropsicología Práctica y Técnicas de Evaluación, Facultad de Psicología (UBA), Argentina. E-mail: mbeizaguirre@gmail.com 4Licenciada en Psicología. Neuropsicóloga en la Clínica de Esclerosis Múltiple, Centro Universitario de Neurología del Hospital Dr. J.M. Ramos Mejía. Facultad de Medicina (UBA), Argentina. E-mail: angeles k84@hotmail.com

5Licenciada en Psicología. Neuropsicóloga en la Fundación Favaloro. Neuropsicóloga en la Clínica de Esclerosis Múltiple, Centro Universitario de Neurología del Hospital Dr. J.M. Ramos

Mejía. Facultad de Medicina (UBA), Argentina. E-mail: cecilia.yas@gmail.com 6Doctora en Psicología. Neuropsicóloga en la Clínica de Esclerosis Múltiple, Centro Universitario de Neurología del Hospital Dr. J.M. Ramos Mejía. Facultad de Medicina (UBA). Profesora Adjunta en la Práctica Profesional Área Clínica, Neuropsicología Práctica y Técnicas de Evaluación, Facultad de Psicología (UBA). Ayudante de primera en la materia Clínica Psicopedagógica, Facultad de Ciencias Sociales, Universidad Nacional de Lomas de Zamora, Buenos Aires,

Argentina. E-mail: svanotti@psi.uba.ar

7Doctor en Medicina. Investigador Independiente del Consejo Nacional de Investigaciones Científicas y Técnicas (CONICET) en el Laboratorio de Deterioro Cognitivo, Hospital Interzonal General de Agudos (HIGA) Eva Perón, San Martín, Buenos Aires. Profesor titular de la cátedra de Neuropsicología, Facultad de Psicología (UBA), Argentina. E-mail: dpolitis@psi.uba.ar

Centro Universitario de Neurología, Hospital Dr. J.M. Ramos Mejía.

Facultad de Medicina, Universidad de Buenos Aires (UBA)

Buenos Aires, Argentina.

\section{Resumen}

Para poder cuantificar e investigar científicamente las quejas mnésicas de los pacientes, se hace necesario contar con un instrumento adaptado y que presente análisis psicométricos adecuados. El objetivo del presente trabajo fue estudiar propiedades psicomé- 
tricas de una versión argentina del Cuestionario de Memoria Prospectiva y Retrospectiva. Para ello se administró el Cuestionario de Memoria Prospectiva y Retrospectiva de forma individual a 192 participantes sanos con más de 27 puntos en el Mini Mental State Examination. La media de edad fue de 39.67 años ( $\mathrm{DE}=12.3)$. La media de escolaridad fue de 14.5 años $(\mathrm{DE}=2.72)$. A un subgrupo de participantes $(n=45)$ denominado Subgrupo A, también se les administraron pruebas de memoria prospectiva y retrospectiva. El Índice Global del Cuestionario presentó una distribución normal. Según el análisis factorial exploratorio se estableció la extracción de un factor que explicó el $35.19 \%$ de la varianza. El coeficiente $\alpha$ de Cronbach fue de .87. La confiabilidad estimada por el método de la división por mitades fue de $.73 \mathrm{y}$ de .84 luego de la corrección con la fórmula de Spearman-Brown. No hubo correlación significativa entre las respuestas del cuestionario y los test de memoria retro y prospectiva en el subgrupo A. Estos resultados permiten concluir que el Cuestionario de Memoria Prospectiva y Retrospectiva presenta adecuados índices de confiabilidad y una estructura de un solo factor. Si bien no se asocia con el rendimiento objetivo en pruebas de memoria, muestra ser apropiado para el registro de las quejas subjetivas de los pacientes en la región de estudio. Palabras clave: memoria; español; memoria prospectiva; memoria retrospectiva.

\section{Abstract}

Memory complaints are the reason for consultation in many cases of neurological injuries and in the majority of aged people who attend the neurological clinic. The presence of memory complaints is a diagnostic criterion for mild cognitive impairment, for which it is necessary to have an appropriate and specific instrument, which would allow us to quantify and scientifically investigate it. The aim of the present study is to analyze the psychometric properties of an Argentine version of the Prospective and Retrospective Memory Questionnaire: the factorial structure of the test; its internal consistency; correlations of the questionnaire with an objective measurement of retro and prospective memory; and correlations of the questionnaire with affective-emotional variables. The Mexican version of the Prospective and Retrospective Memory Questionnaire was administered individually to 192 healthy participants who achieved more than 27 point in the Mini Mental State Examination. A modification was made to the syntax of two sentences in order to increase the comprehensibility. A Global, Prospective and a Retrospective Index of the questionnaire was calculated for each participant. Anxiety (the State-Trait Anxiety Inventory: STAI) and depression (the Beck's Depression Inventory-II) scales were also administered. The mean age was 39.67 years $(\mathrm{SD}=12.3)$. The average of schooling was 14.5 years (SD $=2.72$ ). We also selected a subgroup of participants $(n=45)$ with a mean age of 37.58 years $(\mathrm{SD}=11.4)$, and an average schooling in years of $14.36(\mathrm{SD}=3)$. In addition to the memory questionnaire, this subgroup, called the A Subgroup, was administered prospective (El Cóndor test) and retrospective (The Selective Memory Test) objective memory tests. The mean in the Global Index was 32.25 (SD $=8.49)$, in the Prospective Index it was 17.3 $(\mathrm{SD}=5.01)$ and in the Retrospective Index it was $14.95(\mathrm{SD}=4.07)$. The test for normality of the distribution of the Global Index Kolmogorov-Smirnov (K-S) was $\mathrm{Z}=1.031, p=.238$. In the same way, the other two scores of the questionnaire show a normal curve, K-S of the Prospective Index $Z=1.109, p=.171 ; \mathrm{K}-\mathrm{S}$ of the Retrospective Index $Z=1.264, p=.082$. According to the exploratory factor analysis through the Maximum Likelihood extraction method, a single factor explained $35.19 \%$ of the variance. The Cronbach's $\alpha$ coefficient was .87 . The reliability estimated by the split-half method was .73 and .84 after correction with the Spearman-Brown formula. There was a significant positive correlation of mild degree 
between the Global Questionnaire Index and the STAI Trait, $r=.33, p=.013$, and with the IDB-II, $r=.23, p=.127$. There was no significant correlation between the questionnaire responses and the retro and prospective memory objective tests in the A Subgroup. The Prospective and Retrospective Memory Questionnaire presents adequate reliability indexes and a single factor structure. The results indicate that the questionnaire associates with anxiety and depression in a positive way, but not with the objective measures of retro and prospective memory, in coincidence with the literature about the relationship between cognitive complaints and cognitive tests performance. In sum, this study shows that the questionnaire is an appropriate instrument to quantify patient's memory complaints and particularly for its use in the study region. This study is the first in the country to investigate the psychometric properties of the Prospective and Retrospective Memory Questionnaire, widely used in neuropsychological clinic and research around the world. Efforts should be made to obtain normative data and validation in the pathological population of interest.

Keywords: memory; spanish; prospective memory; retrospective memory

\section{Introducción}

\section{Evaluación de la memoria prospectiva}

La memoria prospectiva (MP) es la capacidad de recordar realizar acciones en el futuro (Ellis, 1996). La característica distintiva de la MP es la autoiniciación (Smith, 2008). Este componente se refiere a la necesidad de que el sujeto inicie por sí mismo el proceso de la evocación y ejecución de una acción planeada previamente, dentro de un periodo de tiempo o a partir de un evento determinado del futuro. Otro aspecto clave de la MP es la identificación o reconocimiento de las señales del medio que indican la necesidad de ejecutar la acción apropiada. De hecho, la MP también ha sido definida como la habilidad de monitoreo del ambiente en busca de los signos que indican la necesidad de realizar la intención planeada (Ellis y Kvavilashvili, 2000); por ejemplo, en el camino al hogar, la imagen de la verdulería evoca comprar frutas.

La MP puede ser evaluada objetivamente a través de tareas en las que se requiere que el examinado recuerde una o varias intenciones demoradas mientras realiza una tarea concurrente de complejidad variada (McDaniel y Einstein, 2000). También puede ser evaluada a través de cuestionarios de autorreporte en los cuales el examinado refleja la presencia de dificultades en la MP en la vida diaria y su frecuencia de aparición.

Otro aspecto de la memoria relacionado con la capacidad de recordar acciones es la memoria retrospectiva (MR), que se define como la capacidad de recuerdo de hechos autobiográficos del pasado. También puede ser evaluada objetiva o subjetivamente. Desde el punto de vista de los paradigmas implementados en investigación, la MP y la MR se diferencian en una serie de características. En las tareas que implican la MR, la atención es explícitamente dirigida por el examinador hacia la recuperación de información almacenada previamente. En cambio, en las tareas de MP el sujeto necesita redirigir la atención desde la tarea de fondo, llamada "actividad concurrente", hacia la intención que, previamente, había formado sin ayuda del examinador (Graf y Uttl, 2001).

Las quejas de memoria son motivo de consulta en muchos casos de lesiones neurológicas y en la mayoría de las personas añosas que concurren a la clínica neurológica, y son criterio de diagnóstico para el deterioro cognitivo leve, por lo cual es necesario contar con un instrumento específico adecuado que permita cuantificarlas e investigarlas científicamente. El Cuestionario de Memoria Prospectiva y Retrospectiva (CMPR; Smith, Della-Sala, Logie y Maylor, 2000) es implementado internacionalmente para evaluar las 
quejas mnésicas de pacientes con daño neurológico. Ha sido validado en países como China (Chan, Qing, Wu y Shum, 2010), Taiwan (Hsu y Hua, 2011), Suiza (Rönnlund, Mäntylä y Nilsson, 2008) y México (González-Ramírez y Mendoza-González, 2011), entre otros. La versión original ha mostrado una estructura de tres factores: el índice de memoria prospectiva (IMP), el índice de memoria retrospectiva (IMR) y un índice global (IG). Esta estructura se basa en la teoría de las quejas mnésicas y se refieren a trastornos relacionados con recordar lo que pasó en el pasado (memoria retrospectiva) o con recordar realizar acciones en el fututo (memoria prospectiva). Es decir que la MP y la MR son constructos diferentes. Pero todas las quejas tienen en común las dificultades en la memoria, es decir que todos los ítems comparten esta característica, por lo que la MP y la MR no son completamente independientes.

La única versión en español publicada es la mexicana (González-Ramírez y Mendoza-González, 2011), que mostró adecuados niveles de consistencia interna en cada una de las subescalas. Sin embargo, la estructura factorial tripartita no pudo ser comprobada, y se halló que un solo factor explicaba mejor la varianza de los puntajes obtenidos. Los autores opinan que la MP y la MR tienen subsistemas cognitivos en común, por lo cual no se encuentran separadas. Dado que es la única versión en español, es potencialmente útil para su uso en población argentina, aunque posee algunos modismos lingüísticos inapropiados para su uso en personas de habla rioplatense. Debido a la discrepancia con los resultados del análisis factorial del estudio original, es necesario realizar investigaciones en la población en la que va a implementarse. ¿Es la versión mexicana del CMPR adecuada para su uso en Argentina?

\section{Revisión de la literatura}

Con respecto a la relación del CMPR con medidas objetivas de MP, se ha encontrado que la correlación es significativa (Kliegel y Jäger, 2006); sin embargo, las quejas prospectivas suelen no asociarse con el rendimiento objetivo en memoria en estudiantes (Uttl y Kibreab, 2011), personas añosas (Mias, Luque, Bastida y Correché, 2015), virus de la inmunodeficiencia humana (VIH; Woods et al., 2007), enfermedad de Parkinson (Foster, McDaniel, Repovš y Hershey, 2009) y enfermedad de Alzheimer (Thompson, Henry, Rendell, Withall y Brodaty, 2015). Lo mismo ocurre con las quejas mnésicas, en general en pacientes con esclerosis múltiple (Demers et al., 2011) y deterioro cognitivo leve (Ryu, Lee, Kim y Lee, 2016). Variables afectivo-emocionales suelen moderar o incidir en la relación entre las quejas cognitivas y la performance objetiva en pruebas neuropsicológicas (Bruce, Bruce, Hancock y Lynch, 2010; Seo, Kim, Choi, Lee y Choo, 2017; Zlatar et al., 2017). Sin embargo, algunos estudios han probado la utilidad de los cuestionarios de quejas subjetivas de memoria en la predicción de la aparición de enfermedad de Alzheimer (Jessen et al., 2010; Silva et al., 2014; Sun, Yang, Lin y Han, 2015; Jessen et al., 2014), por lo cual es importante contar con un instrumento adecuado para recolectar información sobre la memoria subjetiva (Rabin et al., 2015).

\section{Objetivos}

El presente estudio tiene por objetivo general evaluar las propiedades psicométricas del CMPR adaptado para su uso en población de Buenos Aires. Como objetivos específicos se plantean: (a) analizar la estructura factorial del test; (b) explorar su consistencia interna; (c) establecer correlaciones entre el CMPR con una medida objetiva de MP y de MR; (d) analizar la asociación entre el CMPR y variables afectivo-emocionales; y (e) evaluar la relación entre el CMPR y variables sociodemográficas (edad, escolaridad y distribución de género). Se cree que la adaptación tendrá adecuados niveles de consistencia interna y una estructura de un solo factor al igual que 
la versión mexicana. Además, a partir de los resultados de estudios previos revisados, se hipotetiza que los puntajes del cuestionario no correlacionarán con medidas objetivas de memoria prospectiva y retrospectiva, o se hallarán correlaciones muy bajas.

\section{Método}

\section{Diseño}

Estudio de tipo cuantitativo, descriptivo, psicométrico y correlacional.

\section{Participantes}

Se seleccionó una muestra intencional y no probabilística conformada por 192 sujetos adultos de la Ciudad Autónoma de Buenos Aires. El tamaño muestral se estimó en función de la recomendación de utilizar, en la realización de un análisis factorial, una muestra al menos 10 veces mayor que el número de variables o ítems (Nunnally, 1978; Thorndike, 1982). La media de edad de los participantes fue de 39.67 años (DE = 11.4), distribuidos de la siguiente manera: 51 con menos de 30 años, 52 entre 31 y 40 años, 45 entre 41 y 50 años, 39 entre 51 y 60 años y 5 con más de 60 años. La media de escolaridad fue de 13.5 años (DE $=2.72$ ). El $56 \%$ eran mujeres.

Los criterios de inclusión fueron: tener entre 18 y 60 años de edad y más de 7 años de escolaridad. Como criterios de exclusión se consideraron: diagnóstico de enfermedades que puedan causar deterioro cognitivo o impedir la administración de los test y cuestionarios, enfermedad psiquiátrica, antecedentes de alcoholismo o abuso de drogas, obtener menos de 27 puntos en el Mini-Mental State Examination (MMSE; Butman et al., 2001; Folstein, Folstein y McHugh, 1975) y más de 15 puntos en el Inventario de Depresión de Beck (IDB-II; Brenlla y Rodríguez, 2006).

También se seleccionó un subgrupo de participantes (Subgrupo A; $\mathrm{n}=45 ; 71 \%$ mujeres) con una media de edad de 37.58 años $(\mathrm{DE}=11.4)$ y una media de escolaridad en años de $14.36(\mathrm{DE}=3)$. A este subgrupo, además del cuestionario, se le administraron pruebas de memoria prospectiva (El Cóndor; Taussik, 2002) y retrospectiva (Test Selectivo de Memoria y 7/24 Recuerdo Viso-espacial; Cáceres, Vanotti, Rao y RECONEM Workgroup, 2011; Rao, Leo, Bernardin y Unverzagt, 1991).

\section{Instrumentos}

Se confeccionó una entrevista semiestructurada con el objetivo de recabar datos demográficos y clínicos del participante. Para valorar la MP subjetiva se utilizó el CMPR, adaptado al español por González-Ramírez y Mendoza-González (2011), que consta de 16 preguntas que instan al participante a valorar la frecuencia con la que comete errores de memoria. Ocho preguntas se refieren a fallas de MP y se suman para constituir el índice de MP, y ocho se relacionan con fallas retrospectivas que forman el índice de MR. La suma de ambos índices forma el índice global del CMPR. Cada pregunta es contestada con una escala Likert de 5 puntos. Un mayor puntaje indica más quejas de memoria.

Al subgrupo A se les administró, además del CMPR, dos pruebas que evalúan MP y MR objetiva respectivamente, con el objetivo de estudiar la relación entre las quejas subjetivas de memoria y pruebas objetivas de evaluación de la memoria. Para evaluar la MP objetiva se administró el test Cóndor (Taussik, 2002) que consiste en la lectura de un texto acerca de un ave, de contenido emocional neutro, a lo largo de la cual se va refiriendo al sujeto a una lista de instrucciones que se refieren a intenciones de evento y de tiempo que debe realizar sin ayudas durante la tarea de lectura, tanto al finalizarla como luego de una demora con tareas distractoras. Se obtiene un puntaje total de respuestas correctas que consiste en la cantidad de intenciones demoradas realizadas en el momento adecuado (para una descripción más detallada del test ver Cores, Vanotti, Osorio, Garcea y Politis, 2017). 
Para evaluar la MR, se implementó el Test Selectivo de Memoria (TSM) y el 7/24 Recuerdo Viso-espacial, que forman parte de la Batería de Screening Neuropsicológico para esclerosis múltiple (Rao et al., 1991; Cáceres et al., 2011). El TSM consiste en una lista de 12 palabras que se presentan oralmente al examinado en 6 ensayos, quien debe recordarlas libremente en cualquier orden $\mathrm{y}$, luego de un periodo de demora, se le vuelve a pedir que las recuerde. Este test mide adquisición (TSM Aprendizaje) y recuperación de memoria a largo plazo verbal (TSM Recuerdo Diferido). El 7/24 Recuerdo Viso-espacial consiste en el recuerdo, a lo largo de una serie de 5 ensayos, de un diseño de puntos en un tablero de 4 × 6 en el que se ven distribuidos 7 puntos negros cuya ubicación el participante debe recordar para luego reproducirla en un tablero vacío con fichas. Luego de un tablero distractor, se le pide al examinado que vuelva a reproducir de memoria el primer diseño (Recuerdo Inmediato) y nuevamente al cabo de 20 minutos (Recuerdo Diferido).

Además se administraron dos medidas afectivo-emocionales: el State-Trait Anxiety Inventory (STAI), con sus subescalas Estado y Rasgo (Leibovich de Figueroa, 1991; Spielberger, Gorsuch, Lushene, Vagg y Jacobs, 1983), y el IDB-II.

\section{Procedimiento}

En primer lugar, se requirió autorización de uso del test a los autores de la versión original y de la versión mexicana. Para testear la viabilidad de la adaptación mexicana se efectuó un estudio piloto de pequeña escala con estudiantes de grado de la carrera de Psicología $(\mathrm{N}=10)$ en el que, luego de la administración grupal del test, se realizó un debriefing por escrito que preguntaba a los participantes su opinión acerca de las preguntas y sus posibles dificultades de comprensión. Se observó que una pregunta obstaculizaba el entendimiento de los evaluados, por lo que se realizó un cambio de palabras en la versión española-mexicana del CMPR, el cual fue aprobado por los autores de la versión original y mexi- cana ('aún y cuando' por 'aun cuando') y fue exitoso en una prueba piloto posterior en la que se realizó el mismo procedimiento. A continuación, se contactó a los participantes del presente estudio y se les administró la entrevista semiestructurada para recabar datos demográficos y de salud. A cada participante se le administraron las pruebas individualmente bajo la supervisión de una neuropsicóloga entrenada.

\section{Análisis de datos}

Se utilizó estadística descriptiva e inferencial. Se llevó a cabo el análisis psicométrico de la versión española-mexicana del CMPR utilizada en este estudio, para lo cual:

1) Se determinó la validez de constructo mediante el análisis factorial, utilizando el método de Máxima Verosimilitud y rotación Promax. Previamente, para determinar si los datos se ajustaban al análisis factorial, se consideraron los valores obtenidos en la prueba de esfericidad de Bartlett y en el Índice de Kaiser-Meyer-Olkin (KMO). En relación con este último, se decidió que si el índice era mayor a .70 se justificaba el análisis factorial. Para la consideración de un ítem como representativo de un factor se tomó, como criterio, una carga igual o superior a .30 como punto de corte, según las recomendaciones de Tabachnick y Fidell (2013).

2) Se verificó la consistencia interna del test mediante el cálculo del coeficiente $\alpha$ de Cronbach. También se estudió la confiabilidad mediante el método de división por mitades.

3) Se calculó el estadístico Kolmogorov-Smirnov y las medidas de asimetría y curtosis, para estudiar la distribución de los puntajes del cuestionario. Como criterio para evaluar los índices de asimetría y curtosis, se consideraron como excelentes valores entre +1.00 y -1.00 , y adecuados, valores inferiores a +2.00 y -2.00 (George y Mallery, 2011).

4) Se utilizó la correlación $r$ de Pearson para 
estudiar la existencia de asociaciones significativas entre los resultados del CMPR y las medidas de MP y MR, así como con las variables demográficas de edad y años de escolaridad. Además, se utilizó la prueba $t$ de Student de diferencia de medias, con el fin de evaluar la existencia de diferencias significativas en la MP según género.

\section{Resultados}

\section{Estadísticos descriptivos del Cuestionario de Memoria Prospectiva y Retrospectiva}

La media en el Índice Global fue de 32.25 (DE = 8.49), la del Índice de MP fue de 17.3
$(\mathrm{DE}=5.01)$ y la del Índice de MR fue de $14.95(\mathrm{DE}=4.07)$. La prueba de normalidad de la distribución del Índice Global Kolmogorov-Smirnov (K-S) fue de $Z=1.031, p=$ .238. La moda del Índice Global fue de 33 y la mediana de 32. Índice de Asimetría: .354; Curtosis: -.296. Estos datos muestran que la variable Índice Global del CMPR presenta una distribución con una curva normal de los puntajes obtenidos en esta muestra (ver Figuras 1 y 2). De la misma forma, los otros dos puntajes del cuestionario muestran una curva normal (K-S del Índice de MP: $Z=$ $1.109, p=.171$; K-S del Índice de MR: $Z=$ $1.264, p=.082$ ). Los datos percentilares del Índice Global pueden verse en la Tabla 1.

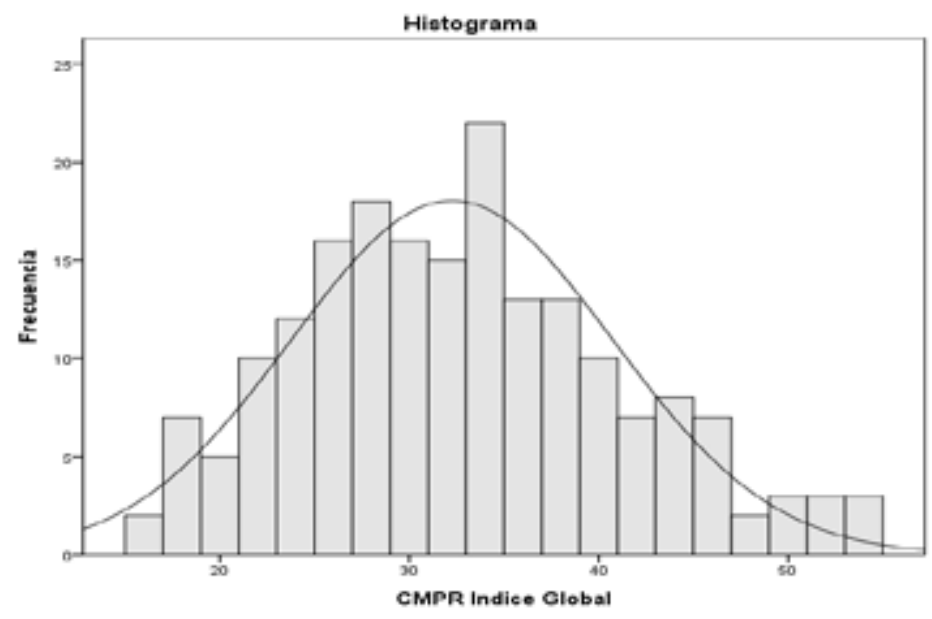

Figura 1. Curva de distribución normal del Índice Global del Cuestionario de memoria. 


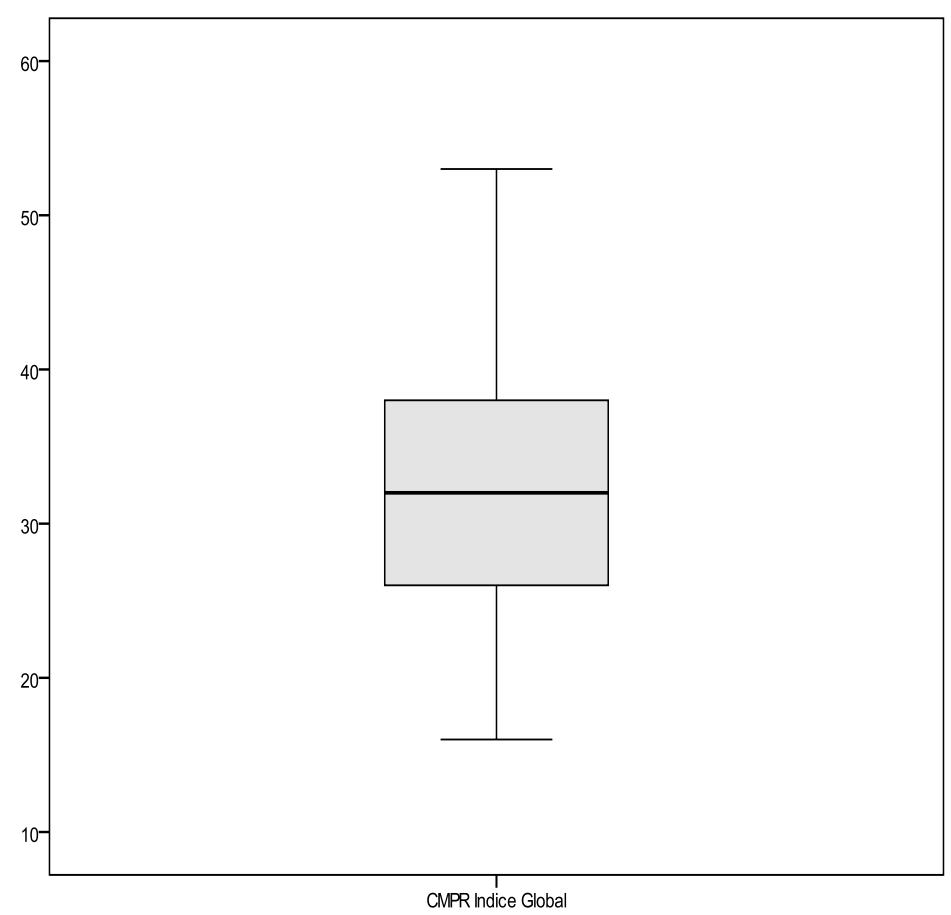

Figura 2. Boxplot de distribución del Índice Global del Cuestionario de memoria prospectiva y retrospectiva.

Tabla 1

Datos percentilares del Índice Global del Cuestionario de Memoria Prospectiva y Retrospectiva.

Percentiles

\begin{tabular}{cccccccc}
\cline { 2 - 7 } & $\mathbf{5}$ & $\mathbf{1 0}$ & $\mathbf{2 5}$ & $\mathbf{5 0}$ & $\mathbf{7 5}$ & $\mathbf{9 0}$ & $\mathbf{9 5}$ \\
\cline { 2 - 7 } Promedio ponderado & 18.65 & 22 & 26 & 32 & 38 & 44 & 48.35 \\
Bisagras de Tukey & & & 27 & 33 & 38 & &
\end{tabular}

Nota: Los percentiles son medidas de posición no central para un conjunto de datos. El percentil para un valor dado indica el porcentaje de datos que son igual o menores que dicho valor; en otras palabras, indica dónde se posiciona una muestra con respecto al total. Por ejemplo, la tabla muestra que el $50 \%$ de los participantes de la muestra presentaron un puntaje de 32 puntos o menos en el CMPR.

Se realizó un análisis factorial exploratorio con los 16 ítems del CMPR a través del método de extracción de Máxima Verosimilitud, con el fin de analizar la correspondencia entre los ítems y el conjunto de factores que pretenden medir (Ferrando y Anguiano-Carrasco, 2010). La medida de adecuación muestral de Káiser-Mayer-Olkin, de .87, y la prueba de esfericidad de Barttlet con valor de $933.879(p \leq .001)$ indicaron la factibilidad de realizar el análisis factorial. Para la extracción de los factores se tomó en cuenta el gráfico de sedimentación (Scree Test) y el análisis paralelo de Horn, para el cual se usó el programa FACTOR v. 8.02 (Lorenzo-Seva y Ferrando, 2011). Se estableció la extracción de un factor que explicó el $35.19 \%$ de la varianza. La Tabla 2 expone los valores obtenidos en el análisis factorial.

El coeficiente $\alpha$ de Cronbach fue de .87 para el Índice Global. La confiabilidad estimada por el método de la división por mitades fue de .73 y de .84 luego de la corrección con la fórmula de Spearman-Brown. 
Tabla 2

Estructura factorial del Cuestionario de Memoria Prospectiva y Retrospectiva.

\section{Items}

Quejas mnésicas

CMPR 10. ¿Planea llevar algo con usted antes de salir de un cuarto, pero minutos después lo deja de lado, aun cuando se encuentra frente a usted?

CMPR 1. ¿Usted decide hacer algo dentro de unos minutos y entonces olvida hacerlo?

CMPR 16. ¿Olvida decir algo a alguien, que tenía la intención de decirle minutos antes?

.652

CMPR 4. ¿Olvida usted algo que le han dicho pocos minutos antes?

CMPR 14. Si trata de comunicarse o contactar a un amigo o pariente y no lo encuentra, ¿olvida tratar de nuevo más tarde?

CMPR 12. ¿Olvida usted llevar un encargo o dar un mensaje que se le había pedido dar a alguien?

CMPR 3. ¿Tiene dificultades para hacer algo que suponía que iba a hacer minutos antes, aun cuando lo que pensaba hacer se encontraba frente a usted, por ejemplo tomar un medicamento o apagar la estufa o la cafetera?

CMPR 7. ¿Olvida comprar algo que había planeado comprar, como una tarjeta de cumpleaños, aun cuando pasa por la tienda?

CMPR 8. ¿Tiene dificultades para recordar cosas que le han pasado los últimos días?

CMPR 11. Pierde algo que acaba de dejar como una revista o unos lentes?

CMPR 5. ¿Olvida usted citas si no le recuerda alguien más sobre ellas o si no utiliza recordatorios como una agenda o un diario?

CMPR 15. ¿Olvida qué vio en televisión el día anterior?

CMPR 9. ¿Usted repite la misma historia a la misma persona en diferentes ocasiones?

CMPR 13. ¿Observa usted algo sin notar que ya lo había visto momentos antes?

CMPR 2. ¿Tiene dificultades para reconocer un lugar que ha visitado antes?

CMPR 6. ¿Tiene usted dificultades para reconocer a un personaje en un programa de radio o televisión de una escena a otra?

Nota: La tabla muestra la carga factorial de cada ítem en el modelo estructural de un factor único. 
Asociación entre el Cuestionario de Memoria Prospectiva y Retrospectiva y las medidas objetivas de memoria prospectiva y retrospectiva

El subgrupo A no se diferenció significa- tivamente del grupo total en edad, escolaridad o distribución de género (Tabla 3). En el subgrupo A, se midió la asociación entre el CMPR con El Cóndor y, por otro lado, con el TSM y el 7/24 Recuerdo Viso-espacial. No se hallaron correlaciones significativas (Tabla 4).

Tabla 3

Comparación en variables demográficas entre la muestra total y el subgrupo A.

\begin{tabular}{cccccc}
\hline & $\begin{array}{c}\text { Total } \\
\mathbf{N = 1 9 2}\end{array}$ & $\begin{array}{c}\text { Subgrupo A } \\
\mathbf{n = 4 5}\end{array}$ & $\mathbf{t}$ & $\mathbf{g l}$ & $\mathbf{p}$ \\
\hline Edad & $39.67(12.3)$ & $37.57(11.4)$ & 1.04 & 235 & .299 \\
Escolaridad & $13.5(2.72)$ & $14.35(3)$ & 1.91 & 235 & .279 \\
\cline { 3 - 6 } & & & $\mathbf{\mathbf { x } ^ { 2 }}$ & $\mathbf{g l}$ & $\mathbf{p}$ \\
\cline { 3 - 6 } Género & $56 \%$ & $71 \%$ & 3.11 & 1 & .054
\end{tabular}

Tabla 4

Correlaciones de Pearson entre el CMPR Índice Global y los test de Memoria Retrospectiva.

\begin{tabular}{ccc}
\hline Test & Puntaje & $\begin{array}{c}\text { CMPR } \\
\text { Índice Global }\end{array}$ \\
\hline \multirow{2}{*}{ TSM } & Aprendizaje & -.19 \\
& Recuerdo Diferido & -.08 \\
$7 / 24$ & Total & -.04 \\
& Recuerdo Inmediato & .01 \\
\multirow{2}{*}{ El Cóndor } & Recuerdo Diferido & -.02 \\
\hline
\end{tabular}

Nota: TSM: Test selectivo de memoria; CMPR: Cuestionario de Memoria Prospectiva y Retrospectiva. En todos los cálculos el nivel de significación fue $p>.05$.

\section{Relación entre el Cuestionario de} Memoria Prospectiva y Petrospectiva y las medidas afectivo-emocionales

A pesar de que el BDI-II se utilizó como criterio de exclusión, por lo cual algunos controles fueron descartados, cuando se analizó la asociación entre el CMPR y el BDI-II se encontró una correlación positiva de grado leve $(r=.23, p=.127)$. Asimismo, el STAI Rasgo correlacionó positiva y significativamente con el CMPR $(r=.33, p=.013)$.
No se halló una correlación significativa con el STAI Estado $(r=.11, p=.467)$.

\section{Asociaciones entre el Cuestionario de Memoria Prospectiva y Retrospectiva y las variables socio-demográficas}

El Índice Global del CMPR correlacionó levemente con la educación en años $(r=-.15$, $p=.038)$. No se observó una correlación significativa con la edad $(r=-.03, p=.632)$.

No se registraron diferencias significativas 
entre hombres y mujeres en el Índice Global del CMPR $(t=-.268, g l=129, p=.789)$.

\section{Discusión}

Los resultados del presente estudio muestran que el CMPR presenta adecuados índices de confiabilidad y una estructura factorial de un solo factor, coincidente con los estudios de la versión mexicana (González-Ramírez y Mendoza-González, 2011), pero no con la versión original inglesa (Crawford et al., 2006; Smith et al., 2000), ni con la suiza (Rönnlund, Mäntylä y Lars-Göran, 2008) ni con la versión taiwanesa (Hsu y Hua, 2011), que han presentado una estructura factorial tripartita.

Al comparar la muestra utilizada para adaptar la versión mexicana (González-Ramírez y Mendoza-González, 2011) con la utilizada en el presente estudio, se observa similar distribución de género y edad promedio. Asimismo, la edad promedio y la distribución por grado de educación de la presente muestra es similar a la implementada para colectar normas de la versión inglesa (Crawford et al., 2006). En cuanto al puntaje promedio obtenido en estas muestra, se registra que la reportada en Crawford et al. (2006) parece ser un poco mayor a la obtenida en el estudio de González-Ramírez y Mendoza-González (2011) y en el presente estudio. Las diferencias en el nivel socioeconómico de las muestras implementadas no medidas podrían explicar el distinto comportamiento del cuestionario en el análisis estadístico factorial.

La presencia de un solo factor en el análisis estructural del cuestionario hallado en el presente estudio podría indicar que la separación entre las quejas mnésicas retrospectivas y prospectivas no es posible, dado que la MP presenta un componente retrospectivo inherente, por lo que en la vida diaria la memoria del pasado y del futuro se experimentan de forma global.

Otro objetivo del presente trabajo consistió en analizar la relación entre el CMPR y las medidas objetivas de MP y MR, así como con variables afectivo-emocionales. Los resultados indican que el CMPR correlaciona con la ansiedad de manera positiva, pero no con las medidas objetivas de MP y MR. Esto se encuentra en divergencia con algunos estudios (Kliegel y Jäger, 2006), pero en convergencia con otros, en los que se ha observado una relación pobre entre el CMPR y la MP objetiva tanto en personas sanas (Mias, Luque, Bastida y Correché, 2015; Uttl y Kibreab, 2011) como en pacientes con daño neurológico (Thompson et al., 2015; Foster et al., 2009). Además, otros cuestionarios de auto-reporte cognitivos han mostrado estar asociados con variables afectivo-emocionales más que con parámetros objetivos neuropsicológicos (Feinstein, 2012; Harvey, Paschall y Depp, 2015; Johnco, Wuthrich y Rapee, 2014). Esto puede deberse a que las personas con alta depresión o ansiedad sobreestiman sus dificultades cognitivas (Stolder, 2012).

Si bien no existe una correlación significativa entre las medidas objetivas y subjetivas de memoria, el CMPR tiene como función, recabar información sobre las quejas mnésicas de los pacientes, las cuales pueden estar influenciadas por diversos factores, entre ellos los afectivo-emocionales como la depresión y la ansiedad (Benedict et al., 2004; Feinstein, 2012). Este estudio halló una asociación entre la ansiedad y las quejas de memoria de los participantes, lo cual refleja la importancia de realizar tanto estudios cognitivos como psicológicos en pacientes que en la clínica neurológica manifiestan la presencia de olvidos en la vida diaria.

Por otra parte, en la presente investigación se analizó la asociación de variables demográficas sobre el CMPR y se encontró que las quejas subjetivas de memoria medidas a través del CMPR están asociadas levemente con la educación, pero no con edad ni el género. Estos datos difieren del estudio de la versión mexicana, el cual mostró una correlación débil con la edad, aunque no reportan análisis de asociación entre las quejas subje- 
tivas de memoria y la educación o el género. En cambio, los resultados se hallan en concordancia con los estudios de la versión original, los cuales no arrojaron asociación significativa con el género o la edad (Smith et al., 2000; Crawford et al., 2006).

En ocasiones, el profesional de la salud interesado en el área cognitiva de su cliente realiza, en su evaluación inicial, algunas preguntas subjetivas al respecto, entre ellas si se olvida las actividades que tiene que hacer o si le cuesta prestar atención. El CMPR es un instrumento útil en la clínica para valorar las quejas mnésicas particulares de cada individuo. Además, es interesante conocer el perfil subjetivo de la memoria de una persona para saber cómo encarar intervenciones en su envejecimiento. La autoeficacia o el autoconocimiento del propio rendimiento influyen en la forma en que las personas lidian con sus errores cognitivos en la vida diaria. Personas añosas sanas, con mayor nivel de quejas mnésicas según los cuestionarios, eligen apoyos para su memoria con mayor nivel de ayuda (Van Etten, Weakley, Schmitter-Edgecombe y Cook, 2016).

Entre las limitaciones del estudio cabe mencionar una muestra reducida compuesta por personas de la región de Buenos Aires. En el futuro, datos normativos deberán desarrollarse a través de una muestra más amplia, compuesta por personas de otras regiones del país, para poder implementar en Argentina este cuestionario en el área clínica. Cabe considerar que para realizar un análisis factorial confirmatorio de la estructura tripartita hallada con la versión original, se requiere una muestra más amplia, en la que quizás los resultados sean sea diferentes. Además, en el presente estudio se implementó la adaptación argentina de la versión mexicana del cuestionario, y no la traducción directa de la versión original, lo cual amerita una comparación de estas versiones en el fututo.

En suma, este estudio es el primero en el país que investiga las propiedades psicométricas del CMPR, un cuestionario de quejas mnésicas ampliamente utilizado en la clínica neuropsicológica e investigaciones alrededor del mundo. La versión presentada es válida y fiable para utilizarla en población argentina para recabar información sobre quejas de memoria. Al igual que otros cuestionarios cognitivos subjetivos no se asocia con medidas de memoria objetiva.

\section{Referencias bibliográficas}

Benedict, R. H. B., Cox, D., Thompson, L. L., Foley, F., Weinstock-Guttman, B. y Munschauer, F. (2004). Reliable screening for neuropsychological impairment in multiple sclerosis. Multiple Sclerosis, 10, 675-678. https://doi.org/10.1191/1352458504ms1098oa

Brenlla, M. E. y Rodríguez, M. (2006). Adaptación Argentina del Inventario de Depresión de Beck-II (BDI-II). En A. T. Beck, R. A. Steer y G. K. Brown (Eds.), BDI-II. Inventario de Depresión de Beck (pp. 11-37). Buenos Aires: Paidós.

Bruce, J. M., Bruce, A. S., Hancock, L. y Lynch, S. (2010). Self-reported Memory Problems in Multiple Sclerosis: Influence of Psychiatric Status and Normative Dissociative Experiences. Archives of Clinical Neuropsychology, 25, 39-48. https://doi.org/10.1093/arclin/ acp092

Butman, J., Arizaga, R. L., Harris, P., Drake, M., Baumann, D., de Pascale, A.... Ollari, J. A. (2001). El ‘Mini-Mental State Examination' en español. Normas para Buenos Aires. Revista de Neurología Argentina, 26, 11-15.

Cáceres, F., Vanotti, S., Rao, S. y the RECONEM Workgroup. (2011). Epidemiological characteristics of cognitive impairment of multiple sclerosis patients in a Latin American country. Journal of Clinical \& Experimental Neuropsychology, First, 1-5. https://doi.org/10.1080/13 803395.2011.603690

Chan, R. C., Qing, Y., Wu, W. y Shum, D. (2010). Prospective memory in healthy Chinese people: The latent structure of the Comprehensive Assessment of Prospective Memory Questionnaire. Neuropsycholo- 
gical Rehabilitation, 20, 459-470. https:// doi.org/10.1080/09602010903425710

Crawford, J. R., Henry, J. D., Ward, A. L. y Blake, J. (2006). The Prospective and Retrospective Memory Questionnaire (PRMQ): Latent structure, normative data and discrepancy analysis for proxy-ratings. British Journal of Clinical Psychology, 45, 83-104. https://doi. org/10.1348/014466505X28748

Cores, E. V., Vanotti, S., Osorio, M., Garcea, O. Politis, G. D. (2017). Prospective memory in patients with relapsing remitting Multiple Sclerosis. Interdisciplinaria, 34(2), 295-306. https://doi.org/10.16888/ interd.2017.34.2.4

Demers, M., Rouleau, I., Scherzer, P., Ouellet, J., Jobin, C. y Duquette, P. (2011). Impact of the cognitive status on the memory complaints in MS patients. Canadian Journal of Neurological Sciences, 38, 728-733. https:// doi.org/10.1017/S031716710005410X

Ellis, J. A. (1996). Prospective memory or the realization of delayed intentions: A conceptual framework for research. En M. A. Brandimonte, G. Einstein y M. A. McDaniel (Eds.), Prospective memory and applications. Mahwah, NJ: Lawrence Erlbaum.

Ellis, J. A. y Kvavilashvili, L. (2000). Prospective memory in 2000: past, present and future directions. Applied Cognitive Psychology, 14, S1-S9. https://doi. org/10.1002/acp.767

Feinstein, A. (2012). Multiple sclerosis and cognitive dysfunction: how accurate are patient's self-assessments? European Journal of Neurology, 19, 535-536. https:// doi.org/10.1111/j.1468-1331.2011.03601.x

Ferrando, P. J. y Anguiano-Carrasco, C. (2010). El análisis factorial como técnica de investigación en psicología. Papeles del Psicólogo, 31, 18-33.

Folstein, M., Folstein, S. E. y McHugh, P. R. (1975). "Mini-Mental State" a practical method for grading the cognitive state of patients for the clinician. Journal of Psychiatric Research, 12, 189-198.
Foster, E. R., McDaniel, M. A., Repovš, G. y Hershey T. (2009). Prospective memory in Parkinson disease across laboratory and self-reported everyday Performance. Neuropsychology, 23, 347-358. https://doi. org/10.1037/a0014692

George, D. y Mallery, P. (2011). SPSS for Windows Step by Step: A simple guide and reference 18.0 update $\left(11^{\circ}\right.$ edition). Boston: Pearson.

González-Ramírez, M. T. y Mendoza-González, M. E. (2011) Spanish version of the prospective and retrospective memory questionnaire (PRMQ-S). Spanish Journal of Psychology, 14, 385-391. https://doi. org/10.5209/rev_sjop.2011.v14.n1.35

Graf, P. y Uttl, B. (2001). Prospective memory: A new focus for research. Consciousness and Cognition, 10, 437-450. https://doi. org/10.1006/ccog.2001.0504

Harvey, P. D., Paschall, G. y Depp, C. (2015). Factors influencing self-assessment of cognition and functioning in bipolar disorder: a preliminary study. Cognitive Neuropsychiatry, 20, 361-371. https://doi. org/10.1080/13546805.2015.1044510.

Hsu, Y. H. y Hua, M. S. (2011). Taiwan version of the prospective and retrospective memory questionnaire: latent structure and normative data. Archives of Clinical Neuropsychology, 26, 240-249. https://doi. org/10.1093/arclin/acr012.

Jessen, F. (2014). Subjective and objective cognitive decline at the pre-dementia stage of Alzheimer's disease. European Archives of Psychiatry and Clinical Neuroscience, 264, 3-7. https://doi.org/10.1007/s00406014-0539-Z

Jessen, F., Wiese, B., Bachmann, C., Eifflaender-Gorfer, S., Haller, F., Kölsch, H.,... y the German Study on Aging, Cognition and Dementia in Primary Care Patients Study Group. (2010). Prediction of dementia by subjective memory impairment: Effects of severity and temporal association with cognitive impairment. Archives of General Psychiatry, 67, 414-422. https://doi. 
org/10.1001/archgenpsychiatry.2010.30.

Johnco, C., Wuthrich, V. M. y Rapee, R. M. (2014). Reliability and validity of two self-report measures of cognitive flexibility. Psychological Assessment, 26, 13811387. https://doi.org/10.1037/a0038009.

Kliegel, M. y Jäger, T. (2006). Can the prospective and retrospective memory questionnaire (PRMQ) predict actual prospective memory performance? Current Psychology: Developmental, Learning, Personality, Social, 25, 182-191. https://doi. org/10.1007/s12144-006-1002-8

Leibovich de Figueroa, N. (1991). Ansiedad: Algunas concepciones teóricas y su evaluación. En M. M. Casullo, N. B. Leibovich de Figueroa y M. Aszkenazi, Teoría y técnicas de evaluación psicológica (pp. 123-155). Buenos Aires: Psicoteca.

Lorenzo-Seva, U. y Ferrando, P. (2011). Manual of the program FACTOR v. 8.02. Recuperado de http://psico.fcep.urv.es/ utilitats/factor/Manual-of-the-FactorProgram.pdf.

McDaniel, M. A. y Einstein, G. O. (2000). Strategic and Automatic Processes in prospective memory retrieval: A multiprocess framework. Applied Cognitive Psychology, 14, S127-S144. https://doi.org/10.1002/ acp. 775

Mias, C. D., Luque, L., Bastida, M. y Correché, M. S. (2015). Quejas subjetivas de memoria, olvidos de riesgo y dimensiones psicopatológicas: aspectos diferenciales entre el declive y deterioro cognitivo leve. Revista Neuropsicología, Neuropsiquiatría y Neurociencias, 15(2), 53-70.

Nunnally, J. C. (1978). Psychometric Theory ( $2^{\text {a }}$ edición). New York: McGraw-Hill.

Rabin, L. A., Smart, C. M., Crane, P. K., Amariglio, R. E., Berman, L. M., Boada, M.,... y Sikkes, S. A. (2015). Subjective cognitive decline in older adults: an overview of self-report measures used across 19 international research studies. Journal of Alzheimer's Disability, 48, Suppl 1, S63-86. https://doi.org/10.3233/
JAD-150154.

Rao, S. M., Leo, G. J., Bernardin, L. y Unverzagt, F. (1991). Cognitive dysfunction in multiple sclerosis. I. Frequency, patterns, and prediction. Neurology, 41, 685-691. https://doi.org/10.1212/wnl.41.5.685

Rönnlund, M., Mäntylä, T. y Nilsson, L. G. (2008). The prospective and retrospective memory questionnaire (PRMQ): Factorial structure, relations to global subjective memory ratings, and Swedish norms. Scandinavian Journal of Psychology, 49, 11-18. $\quad \mathrm{https} / / /$ doi.org/10.1111/j.14679450.2007.00600.X.

Ryu, S. Y., Lee, S. B., Kim, T. W. y Lee, T. J. (2016). Subjective memory complaints, depressive symptoms and instrumental activities of daily living in mild cognitive impairment. International Psychogeriatrics, 28, 487-494. https://doi.org/10.1017/ S1041610215001945.

Seo, E. H., Kim, H., Choi, K. Y., Lee, K. H. y Choo, I. H. (2017). Association of subjective memory complaint and depressive symptoms with objective cognitive functions in prodromal Alzheimer's disease including pre-mild cognitive impairment. Journal of Affective Disorders, 217, 24-28. https://doi.org/10.1016/j.jad.2017.03.062.

Silva, D., Guerreiro, M., Faria, C., Maroco, J., Schmand, B. A. y Mendonça, A. D. (2014). Significance of subjective memory complaints in the clinical setting. Journal of Geriatric, Psychiatry and Neurology, 27, 259-265. https://doi. org/10.1177/0891988714532018.

Smith, G., Della-Sala, S., Logie, R. y Maylor, E. (2000). Prospective and retrospective memory in normal ageing and dementia: A questionnaire study. Memory, 8, 311-321. https://doi. org/10.1080/09658210050117735

Smith, R. E. (2008). Connecting the past and the future. Attention, memory and delayed intentions. En K. Kliegel, M. A. McDaniel y G. O. Einstein (Eds.), Prospective memory. Cognitive, neuroscience, developmental, and applied 
perspectives (pp. 29-52). New York: Lawrence Erlbaum Associates.

Spielberger, C. D., Gorsuch, R. C., Lushene, R. E., Vagg, R. C. y Jacobs, G. S. (1983). Manual for the State-Trait Anxiety Inventory (Form Y). Palo Alto, CA: Consulting Psychologists Press. Stolder, M. E. (2012). Memory self-efficacy in cognitively normal older adults and older adults with mild cognitive impairment (doctoral thesis). University of Iowa. Recuperado de http://ir.uiowa.edu/etd/5063.

Sun, Y., Yang, F. C., Lin, C. P. y Han, Y. (2015). Biochemical and neuroimaging studies in subjective cognitive decline: progress and perspectives. CNS Neuroscience \& Therapeutics, 21, 768-775. https://doi.org/10.1111/ cns. 12395

Tabachnick, B. G. y Fidell, L. S. (2013). Using Multivariate Statistics (6th edition). Boston: Pearson.

Taussik, I. (2002). Desarrollo de un Instrumento para la evaluación de la Memoria Prospectiva (Tesis doctoral inédita). Facultad de Psicología, Universidad de Buenos Aires, Buenos Aires, Argentina.

Thompson, C. L., Henry, J. D., Rendell, P. G., Withall, A. y Brodaty, H. (2015). How Valid Are Subjective Ratings of Prospective Memory in Mild Cognitive Impairment and Early Dementia? Gerontology, 61, 251-257. https:// doi.org/10.1159/000371347

Thorndike, R. L. (1982). Applied Psychometrics. Boston: Houghton-Mifflin.

Uttl, B. y Kibreab, M. (2011). Self-report measures of prospective memory are reliable but not valid. Canadian Journal of Experimental Psychology, 65, 57-68. https://doi.org/10.1037/ a0022843

Van Etten, E. J., Weakley, A., Schmitter-Edgecombe, M. y Cook, D. (2016). Subjective cognitive complaints and objective memory performance influence prompt preference for instrumental activities of daily living. Gerontechnology, 14, 169-176. https://doi.org/10.4017/ gt.2016.14.3.011.00

Woods, S. P., Carey, C. L., Moran, L. M., Dawson, M. S., Letendre, S. L. y Grant, I. (2007). Frequency and predictors of self-reported prospective memory complaints in individuals infected with HIV. Archives of Clinical Neuropsychology, 22, 187-195. https://doi. org/10.1016/j.acn.2006.12.00

Zlatar, Z. Z., Muniz, M., Galasko, D. y Salmon, D. P. (2017). Subjective cognitive decline correlates with depression symptoms and not with concurrent objective cognition in a clinicbased sample of older adults. The Journals of Gerontology. Series B, Psychological Sciences and Social Sciences. Advance online publication. https://doi.org/10.1093/geronb/gbw207.

Recibido: 25 de noviembre de 2018 Aceptado: 27 de agosto de 2020 
